\title{
Immunity and the charismatic camel
}

SCIENTIFIC NAME

Camelus dromedarius

TAXONOMY

PHYLUM: Chordata

CLASS: Mammalia

ORDER: Artiodactyla

FAMILY: Camelidae

\section{Physical description}

The dromedary or Arabian camel is a large, even-toed ungulate with a prominent hump, its most distinctive feature. Sandy brown in color, dromedaries are well adapted to desert conditions, sporting bushy eyebrows, two rows of long eyelashes, hair-lined ears and narrow nostrils that can be closed to protect their facial features from desert sands.

\section{Research résumé}

To complement their characteristic appearance, camels (and related species like llamas and alpacas) also harbor unique antibodies. Camels show strong resistance to many viral and microbial infections, including tetanus, foot-and-mouth disease and mad cow disease (or bovine spongiform encephalopathy) ${ }^{1}$. Their antibodies are several times smaller than those produced by other animals including humans. Dubbed 'nanobodies,' they can enter

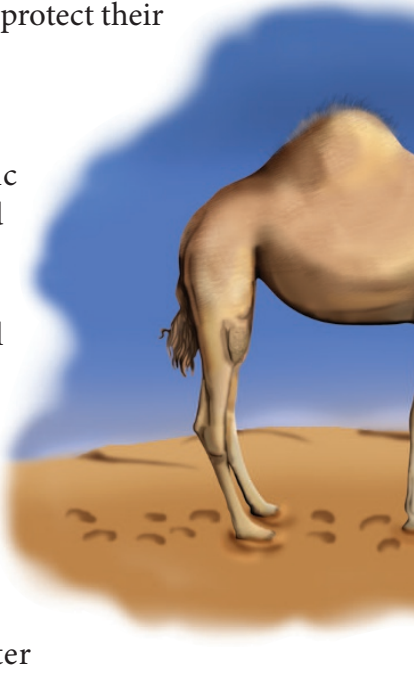
tissues and cells that other antibodies cannot. They can even interfere with enzyme binding sites, contributing to camels' remarkable disease resistance. Their size and ability makes them potentially valuable in biomedical research on a broad range of diseases including cancer, Alzheimer's disease and atherosclerosis.

In one example, researchers used dromedary nanobodies against HER-2, a breast cancer marker, to improve molecular imaging and identification of tumors ${ }^{2}$. The nanobodies are stable and nontoxic and had high visibility, facilitating tumor visualization.

Camelid antibodies may also have applications in brain imaging and in drug transport across the blood-brain barrier. A team of international researchers identified a naturally occurring alpaca nanobody that can cross the blood-brain barrier in vivo under normal physiological conditions and diffuse into brain tissue to reach specific targets ${ }^{3}$. The nanobodies could lead to improved diagnostic and therapeutic techniques for brain diseases such as Alzheimer's disease and brain tumors.

Atherosclerosis is another disease whose diagnosis and treatment could benefit from improved imaging techniques. Investigators recently immunized a dromedary to generate nanobodies targeting VCAM1, an inflammation marker associated with atherosclerosis, that successfully identified atherosclerotic lesions in mice ${ }^{4}$. The technique could someday be used to screen atherosclerotic plaque in people with cardiovascular disease.

Nanobodies are found in camels' blood as well as their milk. Camel milk also contains relatively high levels of insulin, leading some nutritionists to suggest that consumption of camel milk could benefit those with diabetes. In one 16-week randomized study of 54 young adults with type 1 diabetes, fasting blood sugar, hemoglobin A1c levels, serum anti-insulin antibodies and daily

insulin doses were lower in the group that received camel milk in addition to standard treatment administration $)^{5}$. In a separate 2 -year randomized controlled trial of 24 people with type 1 diabetes, those that received camel milk (along with insulin therapy, diet and exercise) had lower levels of blood glucose and hemoglobin A1c and also required smaller insulin doses; 3 of the 12 subjects receiving camel milk required no insulin 6 . Both groups concluded that camel milk improves long-term metabolic control, reducing insulin doses in people with type 1 diabetes.
1. Jassim, S.A.A. \& Naji, M.A. The Desert Ship heritage and science. Biologist 48, 268-271 (2001).

2. Vaneycken, I. et al. Preclinical screening of anti-HER2 nanobodies for molecular imaging of breast cancer. FASEB J. 25, 2433-2446 (2011).

3. Li, T. et al. Cell-penetrating anti-GFAP VHH and corresponding fluorescent fusion protein VHH-GFP spontaneously cross the blood-brain barrier and specifically recognize astrocytes: application to brain imaging. FASEB J. 26, 3969-3979 (2012).

4. Broisat, A. et al. Nanobodies targeting mouse/human VCAM1 for the nuclear imaging of atherosclerotic lesions. Circ. Res. 110, 927-937 (2012).

5. Mohamed, R.H. et al. Camel milk as an adjuvant therapy for the treatment of type 1 diabetes: Verification of a traditional ethnomedical practice. J. Medicinal Food 12, 461-465 (2009).

6. Agrawal, R.P., Jain, S., Shah, S., Chopra, A. \& Agarwal, V. Effect of camel milk on glycemic control and insulin requirement in patients with type 1 diabetes: 2-years randomized controlled trial. Eur. J. Clin. Nutr. 65, 10481052 (2011). 